\title{
Nondestructive Detection of Tilted Planar Flaws on Back Surfaces Using Ultrasonic Wave Interference
}

\author{
Yoshio ARAI $^{* *}$, Marie SATO ${ }^{* * *}$ and Daiki KAWAMOTO ${ }^{* * * *}$ \\ **Saitama University, \\ 255 Shimo-ohkubo, Sakura-ku, Saitama, Saitama 338-8570, JAPAN \\ E-mail: yarai@mech.saitama-u.ac.jp \\ ***Graduate Student of Saitama University, \\ **** Student of Saitama University
}

\begin{abstract}
A new method is proposed to detect a tilted planar flaw on the back surface of a plate using the interference of ultrasonic waves. Parameters for flaw evaluation are developed and examined through experimental results for artificial flaws. The existence of the flaw and its height are estimated by parameter $B_{h}$, which shows the first amplitude of the interference fringe. The effect of tilt angle of the flaw on parameter $B_{\alpha}$, which indicates the amount of asymmetry of the interference fringe along the direction normal to the flaw, is investigated. Flaws larger than $0.5 \mathrm{~mm}$ in height can be detected using the present method with a tilt angle of $20^{\circ}$ from the normal direction of the inspection surface.
\end{abstract}

Key words: Ultrasonics, Nondestructive Flaw Detection, Interference Method, Tilted Planar Flaw

\section{Introduction}

It is important to nondestructively detect flaws nucleated and developed on the inner surface of a structure, such as pressure vessels or pipes, from the outside of the structure to ensure their structural integrity ${ }^{(1)}$. The inspection schedule is based on the resolution limit of flaw detection and sizing of an nondestructive testing method used, as well as the prediction of flaw extension ${ }^{(2)}$. Inclined flaws such as stress corrosion cracks change the reflection efficiency, which is a basic parameter of the amplitude-based sizing approach, when adapting an ultrasonic pulse-echo method for nondestructive inspection ${ }^{(3)}$. The signal-to-noise ratio is the dominant factor in determining the detection limit of the ultrasonic pulse-echo method ${ }^{(4-8)}$. The signal available for measurement by the interference of waves attains a higher signal-to-noise ratio, because random noise does not show interference, and only the interference due to the existence of flaws is measured. A method using this interference of reflected waves could afford significant advantages for enhanced detectability of flaws located on one surface of a plate from another surface. Thus, this ultrasonic interference method for flaw detection offers a promising way to decrease the detection limit of flaw in nondestructive inspection of structures. The present study proposes a new method for detecting and sizing flaws located on the back surface, using ultrasonic interference effects, based on a normal incidence, single transducer, ultrasonic water immersion method. The two-dimensional distribution characteristics of the reflected wave intensity, which is the sum of the waves from the back surface and the flaw, are examined as evaluation criterion for detecting and sizing the flaw. Two kinds of ultrasonic

${ }^{*}$ Received 14 Oct., 2008 (No. 08-0717) [DOI: 10.1299/jmmp.3.518] 
parameters are proposed as criteria, and the availability of the parameters is demonstrated by the experimental results.

\section{Experimental procedures}

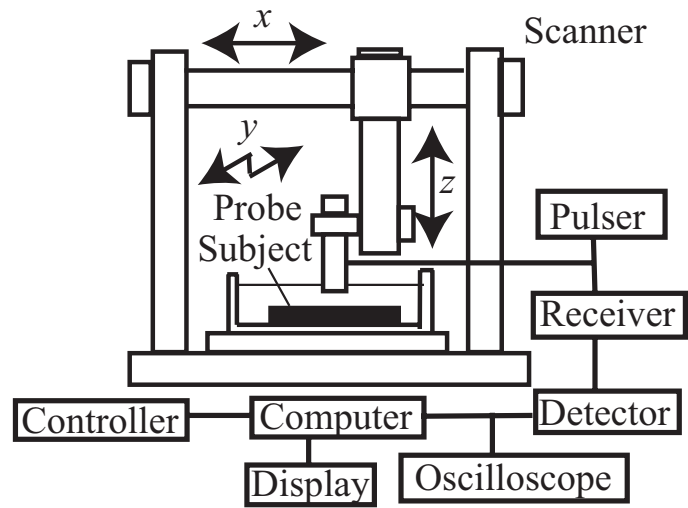

Fig. 1 Configuration of SAM system.

Figure 1 illustrates an ultrasonic measurement system for measuring the two-dimensional distribution of the reflected wave intensity, by mechanically scanning an immersed ultrasonic transducer ("Probe" in Fig. 1) in a plane parallel to the surface of the plate. The immersion method is used because of its high location resolution. For our research and development work, we used a scanning acoustic microscope (Hitachi HSAM 220), although any ultrasonic inspection system with an in-plane scanning mechanism with proper space resolution can be used for the present method. The reflected intensity, $A$, is measured as the maximum amplitude within a gate, which is set for the reflected wave from the back surface and the flaw. $A$ is normalized by $A_{0}$, which is the average reflected intensity measured when no flaw exists on the back surface. The central frequency of the point-focused transducer is $5 \mathrm{MHz}$ (wave length in steel is $1.2 \mathrm{~mm}$ ), its diameter is $10 \mathrm{~mm}$, and the focal length is $20 \mathrm{~mm}$ in water. A plate (structural steel JIS-SS400, with a thickness of $12 \mathrm{~mm}$ ) is in the water bus, as shown in Fig. 1 ("Subject"). The scan pitch is $0.03 \mathrm{~mm}$ square and the measured area is $18 \mathrm{~mm}$ square. The configuration parameters of the artificial flaws introduced on the back surface of the test plate are shown in Fig. 2. The heights, $h$, are $0.5,1.0$, and $2.0 \mathrm{~mm}$, and the tilt angles, $\alpha$, are $0^{\circ}, 5^{\circ}, 10^{\circ}$, and $20^{\circ}$. The flaws prepared by diffusion joining are $50 \mu \mathrm{m}$ wide, while those prepared by electric discharge machining are $250 \mu \mathrm{m}$ which are technically reasonable size and angle to be detected in engineering applications. To develop a detection principle geometrically simple flaws which have these widths of several tens or hundreds wider than the one of cracking flaw in engineering applications are used. Figure 3 shows the cross-sectional view of an artificial flaw machined by electric discharge machining. 


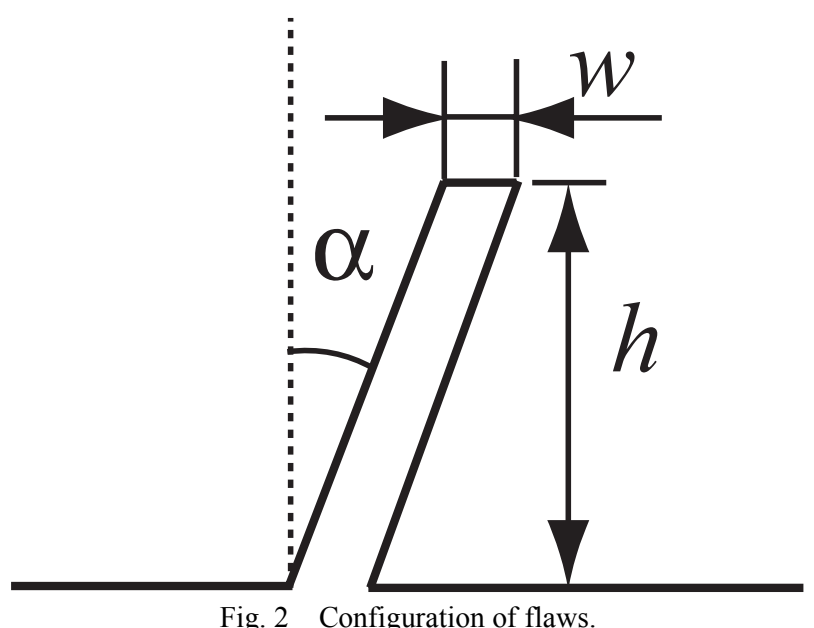

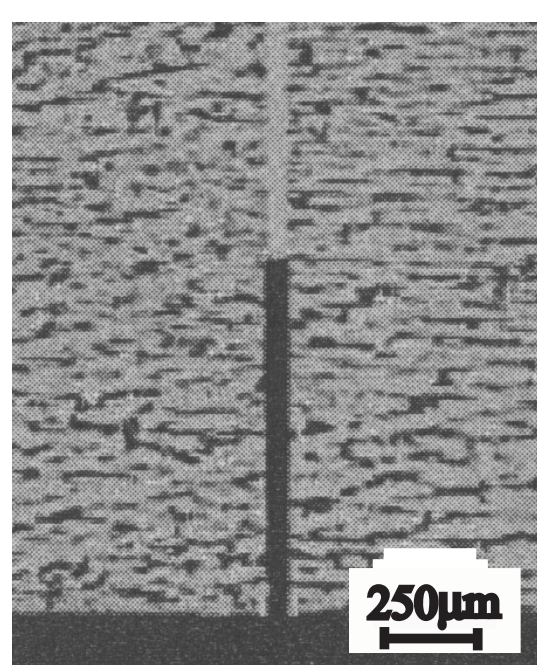

(a) Diffusion joining flaw

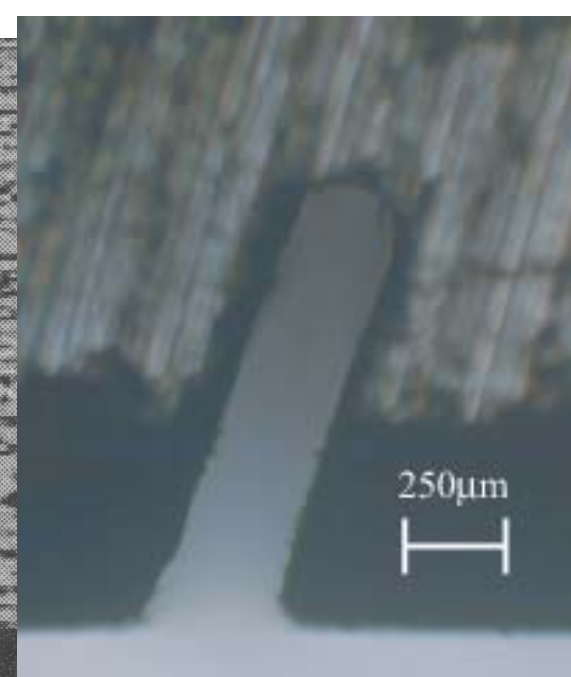

(b) Electric discharge machining flaw

Fig. 3 Cross-sectional shape of slit ( $\mathrm{h}=1.0 \mathrm{~mm}$, (a) $\alpha=0^{\circ}$, (b) $\left.\alpha=20^{\circ}\right)$.

Simulations of wave propagation from the transducer to the reflector (the back surface or the flaw) and backward propagation were conducted to examine the interference mechanism due to the existence of a flaw ${ }^{(9-12)}$. Figure 4 illustrates the simulation model showing the propagation in water, refraction on the water/steel interface, and the reflection from the back surface or the flaw. $c_{1}$ and $c_{2}$ denote the wave velocity in water and the steel, and $d$ is the thickness of the tested plate. $x$ is a coordinate showing the distance from the flaw to the location at the center of the transducer. 


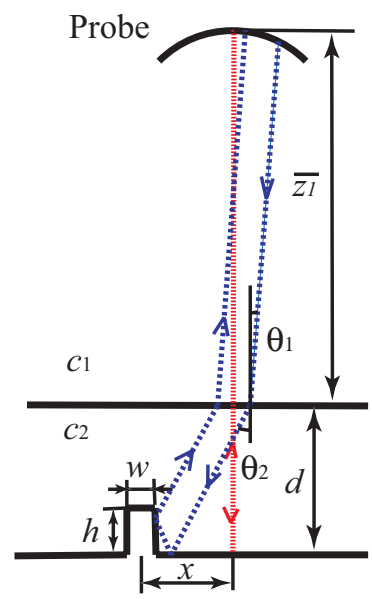

Fig. 4 Simulation model.

Suppose the transducer surface is divided into $\mathrm{n}$ segments. Based on the distributed point source method, the reflected amplitude of a fractional wave generated from the i-th element on the transducer, $f_{i}$, can be given by the following equation:

$$
f_{i}=\frac{a_{i}}{r} \cos \left(\omega t+\phi_{i}\right),
$$

where $a_{i}$ is the initial amplitude, $r$ is the total propagation path, $\omega$ is the angular frequency, and $t$ is the time. $a_{i}$ is assumed to be constant along the transducer surface. The propagation direction of incident wave is assumed to be normal to the probe surface. The refraction is based on the Snell's law and the reflection angle is identical to the incident angle. We only consider the dilatational waves in the specimen.

$$
\phi_{i}=\frac{t_{i}-t_{0}}{T},
$$

where $t_{0}$ and $t_{i}$ are the round trip traveling time of the normal incidence and the incidence from the i-th segment, with the incident angle, $\theta_{i}$, and $T$ is the period of the ultrasonic wave. $r$ and $t_{i}$ can be calculated geometrically depending on the relative location of the transducer from the flaw. The total reflected wave, $F_{n}$, is the sum of the fractional wave from $i=1$ to $\mathrm{n}$.

$$
F_{n}=\sum_{i=1}^{n} \frac{a_{i}}{r} \cos \left(\omega t+\phi_{i}\right)=A_{n} \cos \left(\omega t+\Phi_{n}\right),
$$

where $A_{n}$ and $\Phi_{n}$ are the amplitude and the phase of the total reflected wave, which are given by the following equations:

$$
\begin{aligned}
& A_{i}^{2}=A_{i-1}^{2}+a_{i}^{2}+2 A_{i-1} a_{i} \cos \left(\phi_{i}-\Phi_{i-1}\right), \\
& \Phi_{i}=\tan ^{-1} \frac{A_{i-1} \sin \Phi_{i-1}+a_{i} \sin \phi_{i}}{A_{i-1} \cos \Phi_{i-1}+a_{i} \cos \phi_{i}},
\end{aligned}
$$

where $i=1,2, \ldots, \mathrm{n}$ and $A_{0}$ and $\Phi_{0}$ are zero. $A_{n}$ is the corresponding quantity in simulation to the reflected intensity, $A$, in the experiment.

\section{Experimental results}

The two-dimensional distribution of the reflected wave intensity is shown in Fig. 5, when the height of the flaw is $0.5 \mathrm{~mm}$ and the tilt angle is 0 degrees (normal to the back 
surface). The brightness denotes the reflected intensity at each point in the image. The estimated flaw location is at the center of the image along the vertical direction as shown by the white broken line in Fig. 5. The interference fringes (dark and bright areas) are located symmetrically around and parallel to the flaw. Using the detection of this fringe, small flaws (larger than $0.5 \mathrm{~mm}$ ) are detectable under the experimental conditions (carbon steel, $12 \mathrm{~mm}$ thick, ultrasonic frequency of $5 \mathrm{MHz}$, with a scan pitch of $0.03 \mathrm{~mm}$ ).
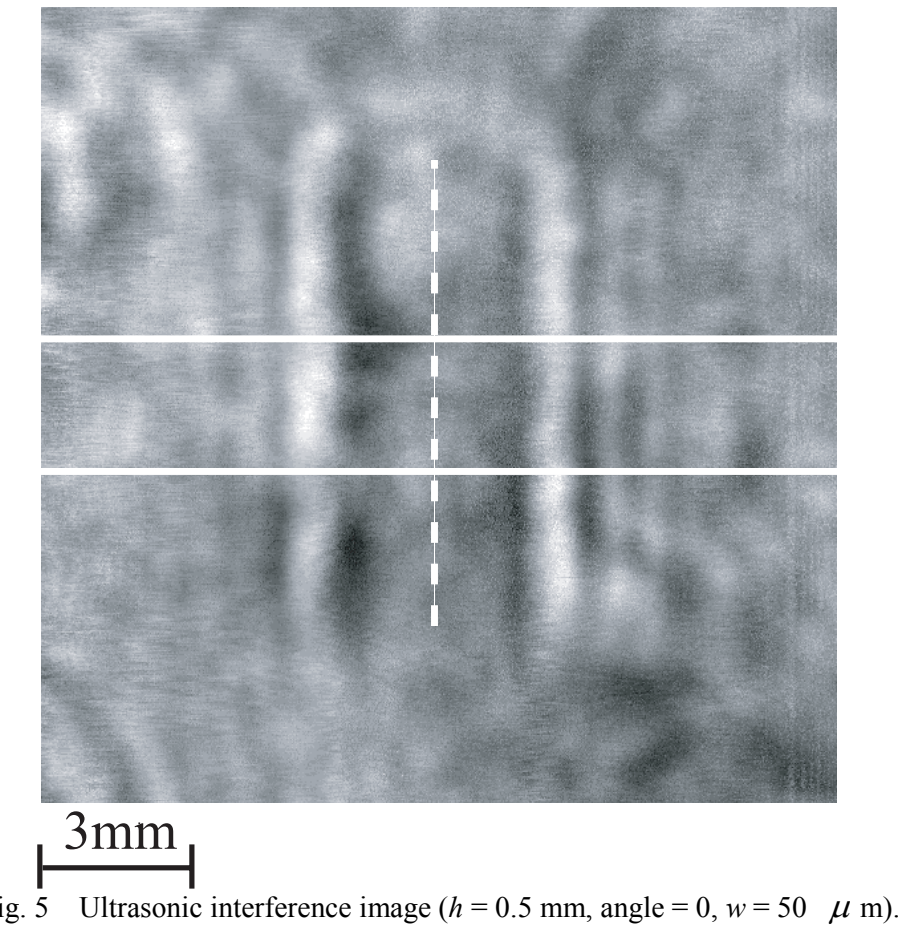

Figure 6 shows the dependence of the distribution of the reflected wave intensity on the height of the flaw. The distributions are the one-dimensional plot of the averaged intensity, at the same distance from the flaw, in the area between the white solid lines shown in Fig. 5, for example, the case where $\alpha=0^{\circ}$. The increase in the height of the flaw, $h$, results in the decrease in the intensity in the negative interference (dark area) and the increase in intensity in the positive interference (bright area). The dependence of the reflected wave intensity distribution on the width of the artificial flaw, $w$, is shown in Fig. 7. A wider flaw has the same effect as an increase in the height of the flaw on the intensity distribution. For the calibration of sizing flaws, applications of a thin artificial flaw, such as the diffusion joining method, gives a larger estimated flaw height, which provides a safer estimation. The inclined flaw gives an asymmetric distribution of the reflected intensity, as shown in Fig. 8, for $\alpha=20^{\circ}$ compared with $\alpha=0^{\circ}$ (normal to the back surface). The reflection on the tilted side faces (left hand side and right hand side) gives different reflection directions because the incident angle to the tilted side face is different side by side. The difference in reflection direction is supposed to be the cause of the asymmetric distribution of the reflected intensity. However the asymmetric distribution can also be seen in the case of thin artificial flaws which are normal to the back surface as shown in Fig. 6. The testing conditions such as normality between the wave incident direction and the back surface should be examined concerning to the symmetric distribution of the reflected intensity in future work. 


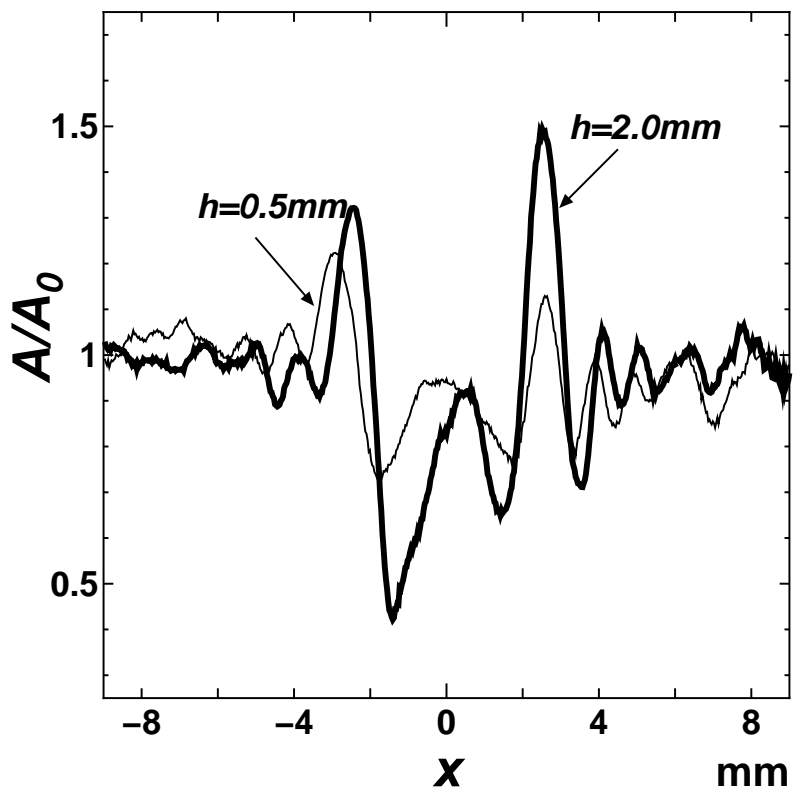

Fig. 6 Dependence of received intensity on height of flaw $\left(h=0.5\right.$ and $2 \mathrm{~mm}, \alpha=0^{\circ}, w=$ $50 \mu \mathrm{m})$.

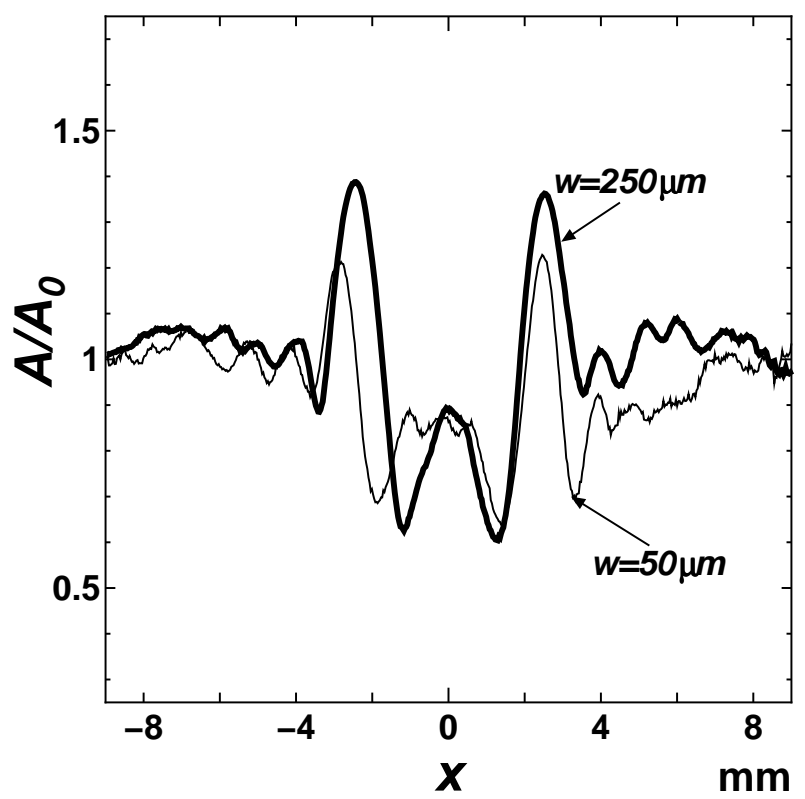

Fig. 7 Dependence of received intensity on width of flaw $\left(h=1.0 \mathrm{~mm}, \alpha=0^{\circ}, w=50\right.$ and $250 \mu \mathrm{m})$. 


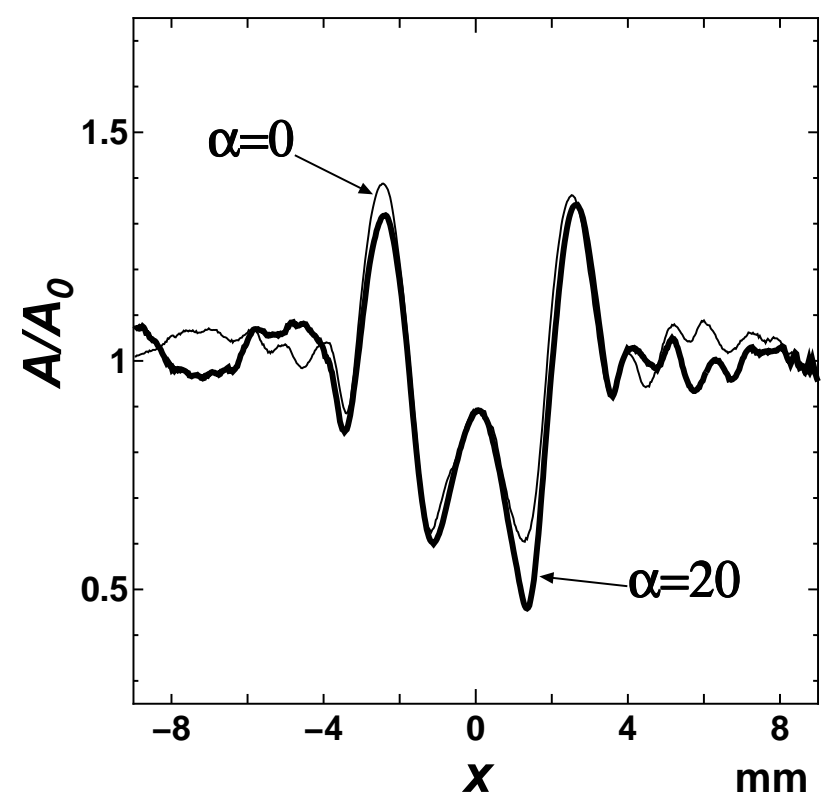

Fig. 8 Dependence of received intensity on inclined angle $\left(h=1.0 \mathrm{~mm}, \alpha=0^{\circ}\right.$ and $\left.20^{\circ}, w=250 \mu \mathrm{m}\right)$.

\section{Discussion}

Based on the simulation results the mechanism of ultrasonic wave interference can be described as follows: When there is no flaw on the back surface, the incident waves come from the central part of the probe (region C in Fig. 9(a)) are reflected on the back surface and return to the ultrasonic probe. The incident angle of the waves generated at a region other than the region $\mathrm{C}$ is too large to return to the probe which means the waves are missed. The offset of the probe location from top of the flaw denoted by $x_{1}$ in Fig. 9 (a) results in the side face reflection of the waves generated at the region $\mathrm{C}^{*}$ (side reflection in Fig. 4) and the occurrence of negative interference between the waves from $\mathrm{C}$ and $\mathrm{C}^{*}$ because of the phase difference ( $\phi_{i}$ in Eq.(2)) occurred due to the increase of propagation path. The increase of offset resulting the propagation path increase larger than one half of the wave length gives positive interference between the waves from $\mathrm{C}$ and $\mathrm{D}$ which is illustrated in Fig. 9 (b). The increase in height of the flaw, h, in Fig. 4 results in the increase in length of the region $\mathrm{C}^{*}$ and $\mathrm{D}$ which magnifies the amplitude of interference fringe. 


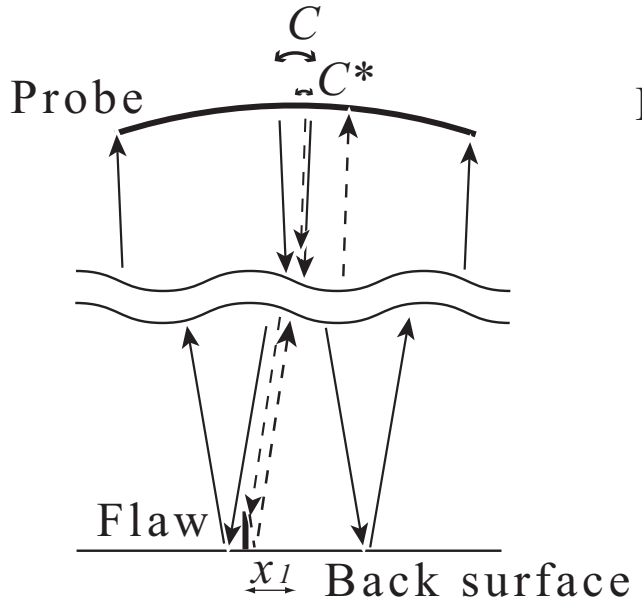

(a) Wave path of negative interference

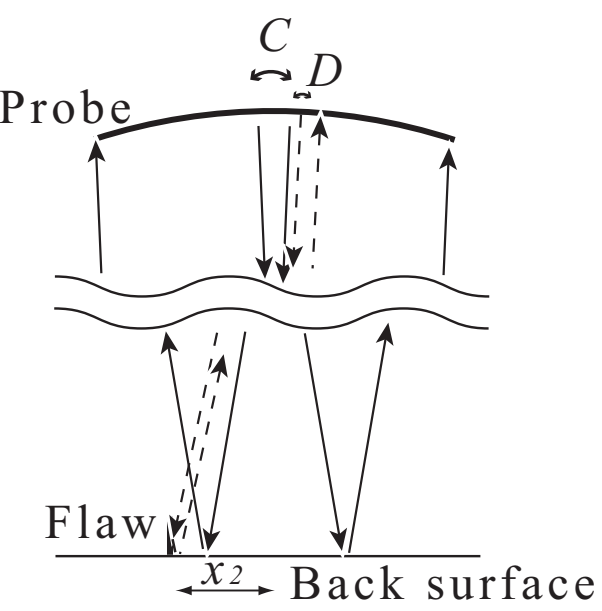

(b) Wave path of positive interference

Fig. 9 Illustration for ultrasonic wave interference.

To characterize the two-dimensional distribution of reflected intensity, the maximum intensities $A_{\max 1}$ and $A_{\max 2}$, and the minimum intensities $A_{\min 1}$ and $A_{\min 2}$ are defined as illustrated in Fig. 10 with typical reflected distribution measured experimentally. $A_{\max }$ and $A_{\min }$ are defined as the average values of $A_{\max 1}$ and $A_{\max 2}$, and $A_{\min 1}$ and $A_{\min 2}$. An ultrasonic parameter to measure the degree of interference due to possible flaws on the back surface, $B_{h}$, is proposed as follows:

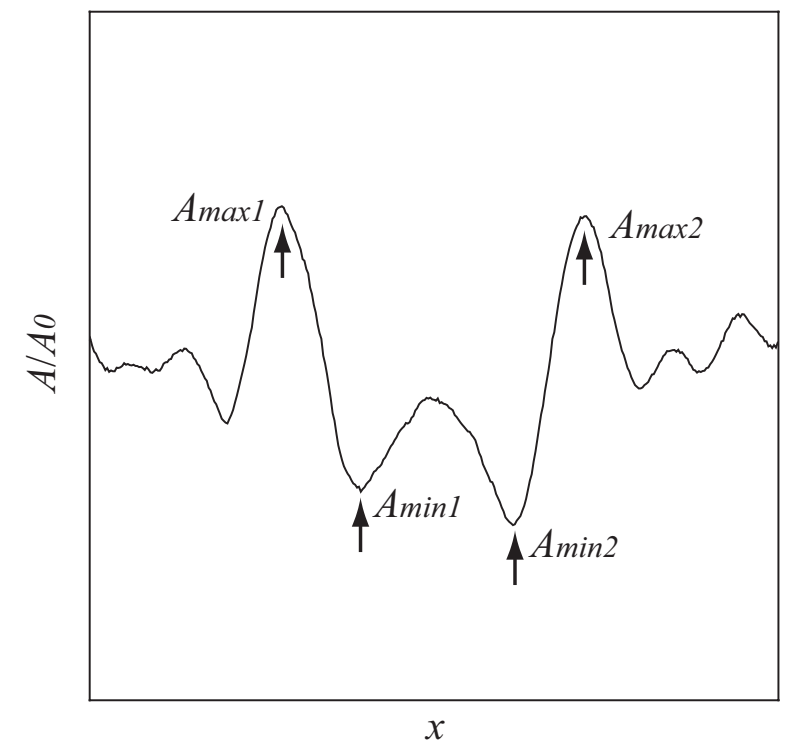

Fig. 10 Definition of $A_{\max }$ and $A_{\min }$.

$$
B_{h}=\frac{A_{\max }-A_{\min }}{A_{\max }+A_{\min }},
$$

where $A_{\max }+A_{\min }$ is used as a reference value which reflects the magnitude of reflection at a location where there is no flaw.

The relationship between $B_{h}$ and the normalized flaw height, $h / \lambda_{2}$, is shown in Fig. 11, where $\lambda_{2}$ is the longitudinal wavelength of the ultrasonic wave in the plate. The broken 
line with "No flaw" denotes the $B_{h}$ level at a location where there is no flaw and the error bar denotes the fluctuation of values in repeated measurements. The experimental results of $B_{h}$, for both diffusion joining and electric discharge flaws, are clearly different from the level for "No flaw" when the flaw height is larger than half of the wavelength. The rate of increase of $B_{h}$ with an increase in flaw height, as predicted by the simulation (full triangles, from 0.06 to 0.09 ), is almost one half of the experimental results (from 0.14 to 0.15). Two dimensional modeling as presented in this study could be the reason for the quantitative discrepancy between the simulation results and the experimental results. The three dimensional simulation which comprises spherical wave as a incident wave and plane flaw as a reflector should be performed in future. The dependence of the $B_{h}-h / \lambda_{2}$ relationship on the flaw width is obvious in the experiment (diffusion joining and electric discharge machining), and a calibration for the specific target flaw (crack-like or hole-like flaw) is necessary to obtain sizing of the flaw. The mechanism to induce the dependence could not be cleared in this study and should be identified in future work.

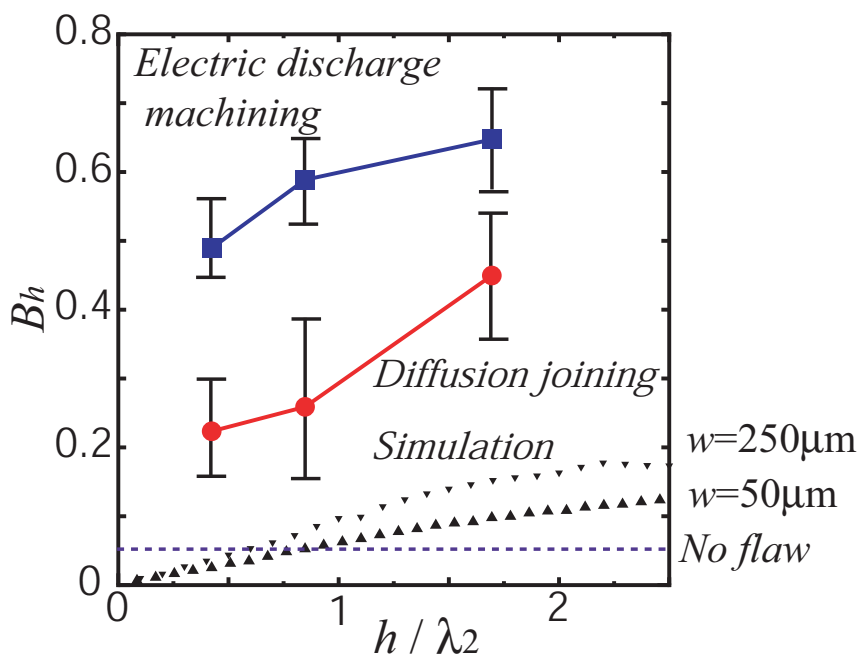

Fig. 11 Influence of flaw height on interference amplitude $\left(\alpha=0^{\circ}\right)$.

The parameter $B_{h}$ shows a weak dependence on the angle of the flaw to the back surface up to $20^{\circ}$, as shown in Fig. 12. Using the ultrasonic interference method, a slant flaw with a $20^{\circ}$ angle and a height larger than $0.5 \mathrm{~mm}$, can be detected using the parameter $B_{h}$ with these measurement conditions.

Asymmetric characteristics of the ultrasonic intensity distributions can be evaluated by the difference in intensity between the maximum and minimum intensities. The average, $B_{\alpha}$, is defined as follows:

$$
B_{\alpha}=\frac{\left|A_{\max 2}-A_{\max 1}\right|+\left|A_{\min 2}-A_{\min 1}\right|}{A_{\max }+A_{\min }},
$$

$B_{\alpha}$ increases with an increase in the tilt angle of the flaw from the normal direction to $20^{\circ}$ as shown in Fig. 12. Comparing the change of $B_{\alpha}$ to the error designated by an error bar in Fig. 12 caused by the testing conditions such as normality between the wave incident direction and the back surface shows obvious dependence on the tilt angle of the flaw. The ultrasonic interference method can measure the tilt angle of the flaw, based on its asymmetric characteristics. Although irregularities of the flaw will affect its asymmetry, in real applications, this method can measure the tilt angle of the planar flaw. 


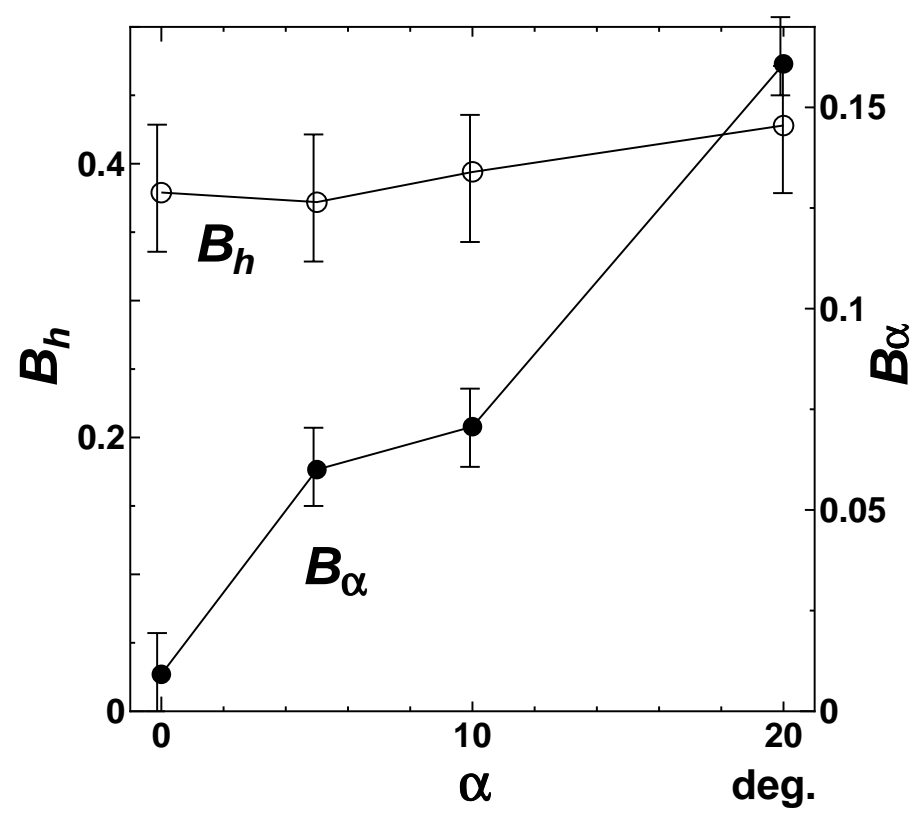

Fig. 12 Influence of tilt angle on $B_{h}$ and $B_{\alpha}$.

\section{Summary}

A new method to detect a tilted planar flaw on the back surface of a plate is proposed using interference of an ultrasonic wave. By detecting the interference fringes, small flaws (larger than $0.5 \mathrm{~mm}$ ) with the tilt angle of $20^{\circ}$ from the normal direction of the plane back surface can be detected under the experimental conditions (carbon steel, $12 \mathrm{~mm}$ thick, with an ultrasonic frequency of $5 \mathrm{MHz}$, and a scan pitch of $0.03 \mathrm{~mm}$ ). Parameters for flaw evaluation are developed and examined through the experimental results of the interference image. The existence of the flaw and its height are estimated by the parameter $B_{h}$, which shows the first amplitude of interference fringe. The experimental results of $B_{h}$ for diffusion joining and electric discharge flaws are clearly different from the level for the results with no flaw when a flaw has its height larger than half of the wavelength. The parameter $B_{h}$ shows a weak dependence on the angle of the flaw to the back surface up to $20^{\circ}$. The effect of the flaw tilt angle on parameter $B_{\alpha}$, which reflects the amount of asymmetry of the interference along the direction normal to the flaw is demonstrated.

\section{References}

(1) Hedden O. F., "Evolution of Section XI of the ASME Boiler and Pressure Vessel Code, " Trans. ASME, J. Press. Vess. Tech., Vol. 122, pp. 234 - 241, 2000.

(2) ASME Boiler and Pressure Vessel Code Section V, 2001, 2003 rev., ASME, New York, 2003.

(3) Vadder, D. D., Azou, P. and Saglio, R., "Determination of orientation and size of badly oriented defects by means of focused probes, " NBS Special Publication 596, Ultrasonic Materials Characterization, eds. H. Berger and M. Linzer, Proc. 1st. Int. Symp. Ultrasonic Mater. Characterization, pp. 11 - 16, 1978.

(4) Moles, M., Dube, N., Labbe, S. and Ginzel, E., "Review of Ultrasonic Phased Arrays for Pressure Vessel and Pipeline Weld Inspections, " Trans. ASME, J. Press. Vess. Tech., Vol. 127 , pp. $351-356,2005$.

(5) Song, L.-M., Qu, X.-H., Xu, K.-X. and Lv L.-N., "Novel SFS-NDT in the field of defect detection, " NDT \& E Inter., Vol. 38, pp. 381 - 386, 2005.

(6) Mayer, K. H., Prestel, W., Weber, D. and Weiss, M., "In-service inspection and defect 
assessment of steam turbine rotors,” Int. J. Pres. Ves. and Piping, Vol. 66, pp. 281 - 292 , 1996.

(7) Lozev, M. G., Spencer, R. L. and Hodgkinson, D., "Optimized inspection of thin-walled pipe welds using advanced ultrasonic techniques," Trans. ASME, J. Press. Vess. Tech., Vol. 127, pp. 237 - 243, 2005.

(8) Lozev, M. G., Smith, R. W. and Grimmett, B. B., "Evaluation of methods for detecting and monitoring of corrosion damage in risers,” Trans. ASME, J. Press. Vess. Tech., Vol. 127, pp. $244-254,2005$.

(9) Achenbach, J. D., Gautesen, A. K. and McMaken, H., "Ray methods for waves in elastic solids," Pitman Advanced Publishing Program, -- (Monographs and studies in mathematics ; 14) 1982.

(10) Tokuoka, T., “Theory of waves,” SAIENSU-SHA, pp. 74, 1984 (in Japanese).

(11) L.W.Schmerr, "A multi Gaussian ultrasonic beam model for high performance simulation on a personal computer," Materials Evaluation, 58(7),pp.882-888,2000.

(12) Placko, D. and Kundu, T., "Modeling of ultrasonic field by distributed point source method," in Ultrasonic nodestructive evaluation, ed. T. Kundu, pp. 143 - 202, 2004. 